\title{
The influence of initial defect morphology of alveolar ridge on volumetric change of grafted bone following guided bone regeneration in the anterior maxilla region: an exploratory retrospective study
}

\author{
Yuan Li, Xiao-Meng Zhang, Shu-Jiao Qian, Shi-Chong Qiao, Hong-Chang Lai, Jun-Yu Shi \\ Department of Oral and Maxillo-facial Implantology, Shanghai Ninth People's Hospital, School of Medicine, Shanghai Jiao Tong University, \\ National Clinical Research Center for Oral Diseases, Shanghai Key Laboratory of Stomatology \& Shanghai Research Institute of Stomatology, \\ Shanghai, China \\ Contributions: (I) Conception and design: JY Shi, HC Lai; (II) Administrative support: HC Lai; (III) Provision of study materials or patients: SJ Qian; \\ (IV) Collection and assembly of data: Y Li, XM Zhang; (V) Data analysis and interpretation: SC Qiao; (VI) Manuscript writing: All authors; (VII) \\ Final approval of manuscript: All authors. \\ Correspondence to: Jun-Yu Shi; Hong-Chang Lai. Department of Oral and Maxillo-facial Implantology, Shanghai Ninth People's Hospital, School of \\ Medicine, Shanghai Jiao Tong University, 639 Zhizaoju Road, Shanghai 200011, China. Email: Sjy0511@hotmail.com; 1hc9@hotmail.com.
}

Background: This study aimed to explore the influence of initial ridge defect morphology on the outcome of guided bone regeneration (GBR) in the anterior maxilla region.

Methods: Cone beam computed tomography (CBCT) examinations of patients who participated in a previous randomized controlled trial were used to assess linear and volumetric changes of bone grafts (LCB and VCB) from immediately (T0) to 6 months (T1) after surgery. The three-dimensional (3D) surface rendering of the initial defect was reconstructed, and morphological variables were defined in mesial-distal, buccal-lingual, and coronal-apical directions. The Spearman correlation, logistic regression model, and receiver operating characteristic (ROC) analyses were used to assess the possible association between initial defect morphological variables and VCB.

Results: A total of 62 eligible patients were included in this study. The median value of LCB was less than $20 \%$ at different levels, while the corresponding value of VCB was $52.0 \%$. The Spearman correlation analysis showed that the standard deviation of buccal-lingual distance $\left(\mathrm{BL}_{\mathrm{SD}}\right)$ was negatively associated with $\operatorname{VCB}(\mathrm{r}=-0.315, \mathrm{P}=0.013)$, whereas the ratio of maximum coronal-apical/mesial-distal distance $\left(\mathrm{R}_{\mathrm{mCA} / \mathrm{mMD}}\right)$ was positively related to $\operatorname{VCB}(\mathrm{r}=0.607, \mathrm{P}<0.001)$. The multivariate regression analysis revealed that the prognosis effect of BLSD (OR: 0.220, 95\% CI: 0.074 to $0.655, \mathrm{P}=0.0047$ ) and $\mathrm{R}_{\mathrm{mCA} / \mathrm{mMD}}(\mathrm{OR}: 7.045,95 \%$ CI: 2.361 to $21.024, \mathrm{P}=0.0017$ ) remained significant. ROC curve analysis showed that $\mathrm{R}_{\mathrm{mCA} / \mathrm{mMD}}$ could be used to correctly classify VCB in $78.9 \%$ patients and BLSD in $71.0 \%$ of patients, as classified by the median of VCB. The discrimination value of BLSD and $\mathrm{R}_{\mathrm{mCA} / \mathrm{mMD}}$ revealed the areas under curve (AUC) of 0.71 (95\% CI: 0.545 to 0.883 ) and 0.74 (95\% CI: 0.573 to 0.913 ), respectively.

Conclusions: Within the limitations of this study, the present data confirmed the effect of initial ridge morphology on the GBR outcome in the anterior maxilla region. Specifically, a defect morphology with more BLSD and/or lower $\mathrm{R}_{\mathrm{mCA} / \mathrm{mMD}}$ may significantly decrease the resorption amount of grafted bone.

Keywords: Initial ridge defect morphology; guided bone regeneration (GBR); cone beam computed tomography (CBCT); morphological contour interpolation (MCI)

Submitted Feb 08, 2020. Accepted for publication Sep 14, 2020.

doi: 10.21037/atm-20-1432

View this article at: http://dx.doi.org/10.21037/atm-20-1432 


\section{Introduction}

Prosthetic-driven implant placement is often compromised by insufficient alveolar bone volume. Various methods have been applied to compensate for alveolar bone defects, such as guided bone regeneration (GBR), bone splitting, block bone grafting, and distraction osteogenesis (1-3). Among these methods, GBR is the most well-documented clinical procedure to augment alveolar bone in the localized bone defect (4-6).

As the most widely used method for horizontal bone augmentation, the outcomes of GBR procedures vary in the literature. Several studies demonstrated the mean horizontal bone gain after GBR ranged from 3.0 to 5.0 millimeter (mm) $(7,8)$, while Urban and his colleagues reported that more than $7.0 \mathrm{~mm}$ horizontal bone gain could be achieved (9). Several factors, such as barrier membrane, bone substitute, wound closure, and initial defect morphology, are related to the outcomes of GBR procedure $(10,11)$.

Over the past three decades, the influence of barrier membranes and bone substitute on GBR outcome has been well studied (12). The desirable characteristics of barrier membranes utilized for the GBR procedure include biocompatibility, cell occlusion properties, integration by the host tissues, clinical manageability, and space-making ability. Favorable bone substitute requires osteogenic, osteoinductive, and osteoconductive properties. With regard to wound closure, a passive tension flap would benefit wound healing. Some previous studies $(13,14)$ pointed out that some compressive forces during wound closure could result in membrane collapse and displacement of the bone substitute which had a significant impact on the outcome of GBR.

Indeed, initial defect morphology also plays an important role in bone regeneration. Favorable defect morphology could benefit the stability of grafting biomaterials, while unfavorable defect morphology usually requires additional stabilizing accessory, such as a fixture pin or tenting screw. Some clinicians have tried to establish classification systems to define the initial defect morphology and predict the treatment outcomes. So far, several classification systems have been proposed. One classification was established based on the alveolar bone width (Class A: bone width $>5 \mathrm{~mm}$, Class B: $2.5 \mathrm{~mm}<$ bone width $<5 \mathrm{~mm}$, Class C, and D: bone width $<2.5 \mathrm{~mm}$ ) (15). Small to moderate horizontal bone loss of 3 to $6 \mathrm{~mm}$ in the horizontal, vertical, and combination (HVC) classification was considered as a good indication for GBR treatment (16). In addition, a recent study reported an $\mathrm{ABC}$ classification of alveolar ridge vertical atrophy (17). These classifications indicated implicitly that the ridge morphology may influence the bone augmentation outcome. However, none of these classifications could be quantitatively correlated with the alteration of grafted bone, which meant that information about the prognostic value of initial defect morphology on the GBR outcome was limited.

Cone-beam computerized tomography (CBCT) is widely used for pre-surgical assessment of surgical anatomy and measurement of the healing process of augmented tissue. For pre-surgical assessment, three-dimensional (3D) reconstruction of initial bone defect could be obtained using a mirror image of the unaffected side in the pre-operative image, which was a routine process of the reconstruction of craniomaxillofacial defects (18). For post-operative assessment, only two-dimensional linear measurement or marginal bone loss were used to assess GBR outcome in the majority of studies (19), though a recent study by Tonetti et al. had demonstrated the overall diagnostic accuracy of CBCT was high (20). In our previous study (21), a novel semiautomatic segmentation method has been demonstrated to be highly reliable to segment and reconstruct the grafted bone following GBR. With this method, the volumetric measurement could be achieved, and 3D changes of augmented bone could be visually observed.

Thus, using the 3D analysis method, this study aimed to explore the influence of the initial defect morphology of alveolar ridge on volumetric change of grafted bone following the GBR procedure in the anterior maxilla region. We present the following article in accordance with the STROBE reporting checklist (available at http://dx.doi. org/10.21037/atm-20-1432).

\section{Methods}

\section{Study design and population}

The present study was designed as an exploratory retrospective study, and the medical data of eligible patients were retrieved from a randomized controlled trial (ChiCTRIPR-15006885) conducted in the Department of Oral and Maxillo-facial Implantology, Shanghai Ninth People's Hospital from 2016 to 2018. The study conformed to the provisions of the Declaration of Helsinki (as revised in 2013). The study protocol was approved by the Ethics Committees of Shanghai Ninth People Hospitals [China, No. (2015)48] and informed consent was taken from all the patients. 


\section{Inclusion criteria}

(I) Patients had single tooth loss in the anterior maxilla for at least 3 months; (II) patients needed implant placement and simultaneous GBR; (III) patients took CBCT examinations immediately and 6 months after surgery; (IV) patients had uneventful healing.

\section{Exclusion criteria}

(I) Patients had an obvious vertical bone defect; (II) patients had wound exposure and postoperative infection; (III) heavy smoker (>10/day); (IV) patients had a history of periodontitis.

\section{Surgical procedure}

All treatment was operated by an experienced surgeon (HC Lai). Mid-crest incision combined with intra-sulcular and vertical incisions were made under local anesthesia, and a mucoperiosteal flap was raised. Straumann Bone Level SLA implants (Institute Straumann AG, Waldenburg, Switzerland) were placed according to the manufacturer's instructions. A natural bovine bone matrix composed of hydroxyapatite and collagen (Zhenghai Biotechnology, particle size $0.25-1 \mathrm{~mm}$, Shandong, China) covered with a control membrane (Bio-Gide ${ }^{\circledR}$, Geistlich AG, Wolhusen, Switzerland) or a test bovine dermis-derived collagen membrane (Zhenghai Biotechnology, Shandong, China) were used to augment the horizontal bone defect. Overcontour protocol was conducted by putting biomaterials beyond the surrounding native bone by $2 \mathrm{~mm}$. The wound was closed with Ethilon 5-0 nylon sutures in a tension-free approach. All patients were prescribed antibiotics for 3 days. The patient was instructed to use chlorhexidine oral rinse $(0.12 \%)$ for $60 \mathrm{~s}$ five to six times a day for 14 days. Sutures were removed 10-14 days after surgery.

\section{CBCT data acquisition}

All imaging scans were acquired with a CBCT machine (i-CAT, Imaging Sciences International, Hatfield, PA, USA) in the Department of Radiology, Shanghai Ninth People's Hospital, Shanghai Jiao Tong University School of Medicine. CBCT examinations were performed immediately after surgery (T0) and 6 months later (T1). The imaging parameters were set at a tube voltage of $120 \mathrm{kV}$, tube current of $5 \mathrm{~mA}$, voxel resolution of $0.25 \mathrm{~mm}$, and field of view (16 cm diameter $13 \mathrm{~cm}$ height) for a scan time of 20 seconds. Data from the scans were saved in the Digital Imaging and Communications in Medicine format.

\section{Outcomes}

Two examiners (JY Shi, Y Li) performed all measurements in Medraw ${ }^{\circledR}$ software. Ten randomly selected CBCT images were used to test the intra-observer reliability of CBCT measurements. The intraclass correlation coefficient (ICC) for linear measurement and volumetric measurement was 0.92 and 0.95 (95\% CI: 0.90-0.97), respectively, indicating high intra-examiner agreement.

\section{Linear measurement}

For each patient, a cross-sectional image at T0 and T1, taken as closely as possible through the implant center, was selected, and saved so that two examiners could perform the measurements on identical slices. The thickness of the facial bone wall (TBW) was measured (in $\mathrm{mm}$ ) with Image J software at 3 different levels: 2,4 , and $6 \mathrm{~mm}$ apical to the implant shoulder. The known implant length was used for post-calibration measurements and the calibration formula was true $\mathrm{TBW}=$ (known implant length/measured implant length) $\times$ measured TBW. Linear change of bone grafts (LCB) was calculated as $\mathrm{TBW}_{\mathrm{T} 0}-\mathrm{TBW}_{\mathrm{T} 1} / \mathrm{TBW}_{\mathrm{T} 0} \times 100 \%$.

\section{Volumetric measurement}

A novel semi-automatic segmentation protocol based on morphological contour interpolation (MCI) algorithm was used to segment the bone graft from surrounding native bone and soft tissue. Our previous study described the details of the novel protocol (21). Briefly, the obtained DICOM files of CBCT images were imported into Medraw ${ }^{\circledR}$ software (Image Medraw Technology Co., Ltd, Shanghai, China). The segmentation procedure mainly consisted of two steps. In the first step, manual drawing was performed to trace the perimeters of the bone graft area on coronal slices without or with few artifacts' interferences. Manually segmented slices were selected as the input slice of the MCI algorithm. In the second step, maximal inter-region overlaps achieved by heuristic alignment based on the minimal displacement were performed prior to inter-slice interpolation between corresponding regions. Afterward, the iteration of the MCI process automatically computed 


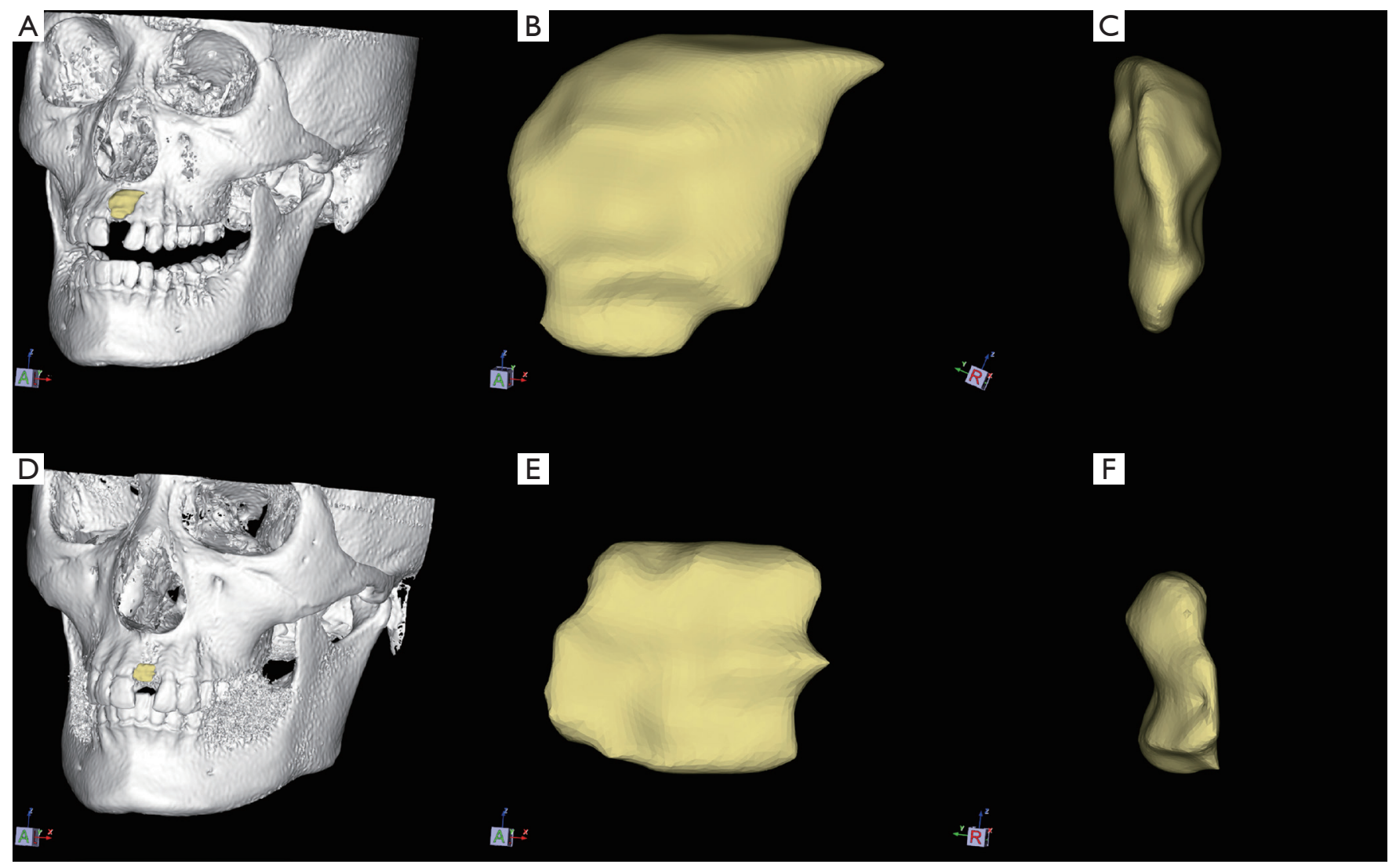

Figure $13 \mathrm{D}$ surface rendering of bone grafts at T0 (A) and T1 (D) were reconstructed based on original CBCT data. The change of the whole bone grafts could be visible. (B,E) frontal view of bone grafts at T0 and T1; (C,F) lateral view of bone grafts at T0 and T1.

a transition sequence between a pair of corresponding input slices. 3D surface rendering of bone graft could be visualized in a separate $3 \mathrm{D}$ visualization window (Figure 1).

After segmentation, the volume of bone graft (VBG) at T0 and T1 was calculated by the built-in statistics module based on segmented voxel size and quantity. The volumetric change of bone grafts (VCB) was calculated as $\mathrm{VBG}_{\mathrm{T} 0}$ $\mathrm{VBG}_{\mathrm{T} 1} / \mathrm{VBG}_{\mathrm{T} 0} \times 100 \%$.

\section{Initial defect morphology definition}

The 3D surface rendering of the initial defect was obtained by using a mirror image of the contralateral unaffected alveolar ridge. The following variables were used to define the initial defect morphology of alveolar ridge (Figure 2):

(I) The mesial-distal distance of initial bone defect (MD): After reconstruction, the initial bone defect was divided into slices in $0.25 \mathrm{~mm}$ from mesial to distal (S1, S2 to Sn) at the sagittal plane. The number of slices at the sagittal plane (N.S) was counted and the maximum MD value (mMD) was calculated for each patient.

(II) The coronal-apical distance of initial bone defect (CA): After reconstruction, the initial bone defect was divided into slices in $0.25 \mathrm{~mm}$ from coronal to apical (H1, H2 to Hn) at the horizontal plane. The number of slices at the horizontal plane (N.H) was counted and the maximum $\mathrm{CA}$ value (mCA) was calculated for each patient.

(III) The buccal-lingual distance of initial bone defect (BL): Intersection points of slices in sagittal and horizontal planes on the outer surface of $3 \mathrm{D}$ surface rendering were marked and projected onto the inner surface. The distance of projection lines was recorded, and maximum $\mathrm{BL}$ value $(\mathrm{mBL})$, the mean value of $\mathrm{BL}\left(\mathrm{BL}_{\mathrm{Mean}}\right)$, and the standard deviation of $\mathrm{BL}\left(\mathrm{BL}_{\mathrm{SD}}\right)$ were calculated for each patient.

For all the included patients, the following demographic 


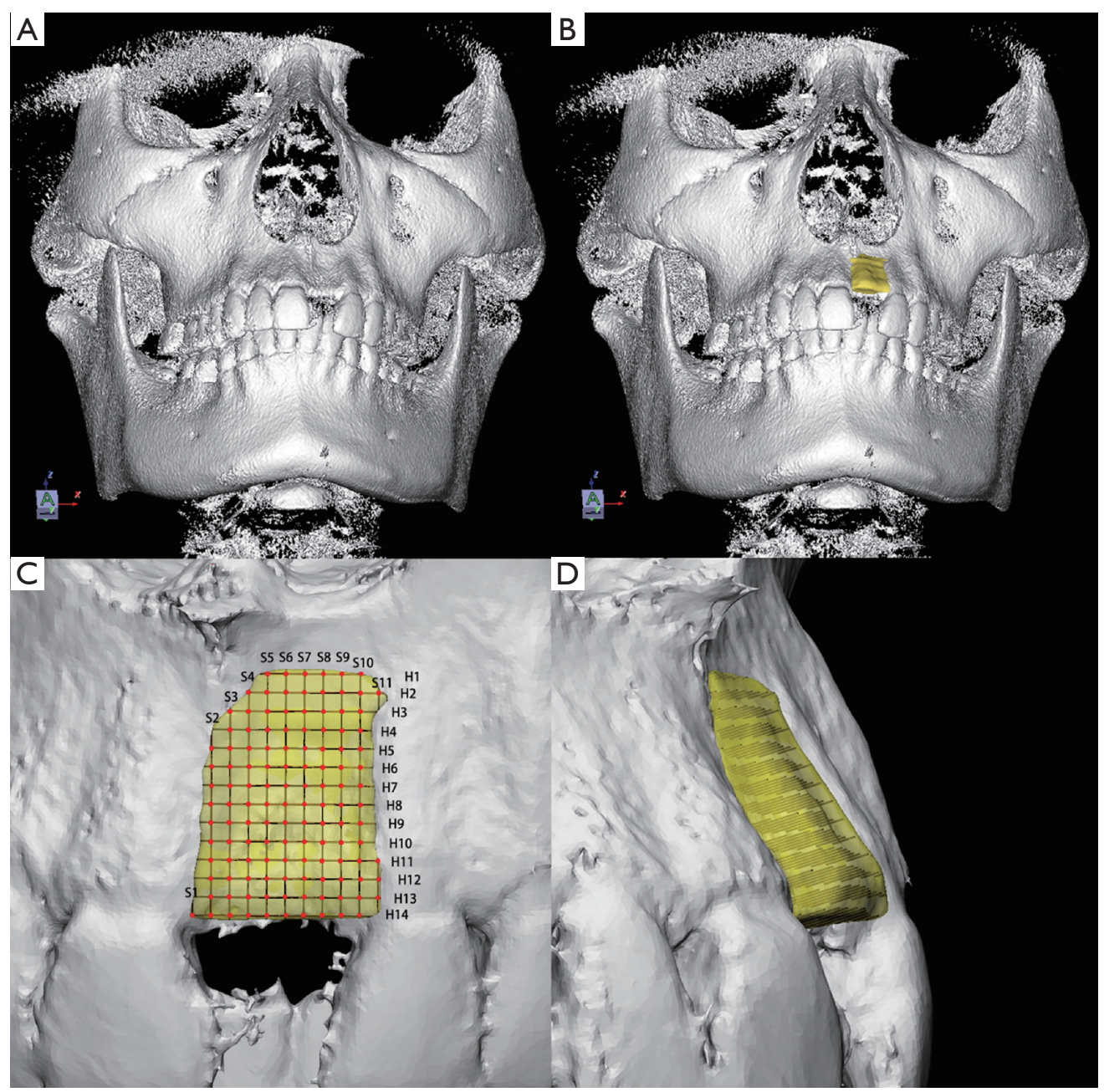

Figure 2 The initial bone defect was reconstructed, and the initial defect morphology was defined on mesial-distal, coronal-apical, and buccal-lingual dimension. (A) Preoperative CBCT reconstruction image; (B) 3D surface rendering of initial bone defect obtained using a mirror image of the contralateral alveolar ridge; (C) 3D surface rendering of initial bone defect was divided into slices in $0.25 \mathrm{~mm}$ from mesial to distal (S1, S2 to Sn) at the sagittal plane and from coronal to apical (H1, H2 to Hn) at the horizontal plane. Intersection points of slices in sagittal and horizontal planes were marked on the outer surface of 3D surface rendering; (D) intersection points on the outer surface were projected onto the inner surface. The length of projection lines was considered as the buccal-lingual distance of bone defect.

and initial defect morphological data were collected: gender; age; type of membrane; implant site; the mean value of $\mathrm{BL}$ $\left(\mathrm{BL}_{\text {Mean }}\right) ; \mathrm{BL}_{\mathrm{SD}} ; \mathrm{mCA} ; \mathrm{mMD} ; \mathrm{mBL}$; ratio of $\mathrm{mCA} / \mathrm{mMD}(\mathrm{R}$ $\mathrm{mCA} / \mathrm{mMD})$; ratio of $\mathrm{mCA} / \mathrm{mBL}\left(\mathrm{R}_{\mathrm{mCA} / \mathrm{mBL}}\right)$ and ratio of $\mathrm{mBL} /$ $\mathrm{mMD}\left(\mathrm{R}_{\mathrm{mBL} / \mathrm{mMD}}\right)$.

\section{Statistical analysis}

Data were analyzed using the statistical software Statistical Package for Social Science (SPSS), version 25 (SPSS, Inc.; IBM Corporation). Categorical variables are expressed as frequencies and percentages. Continuous variables were described as mean \pm standard deviation $(\mathrm{SD})$ or medians [interquartile ranges (IQRs)] for non-normal distribution. The normality of distributions was assessed by the ShapiroWilk test. The Spearman correlation coefficient was calculated between baseline demographic, initial defect morphological variables, and VCB. Subsequently, patients were classified into two groups according to the median of VCB. The association of baseline demographic, initial defect morphological variables with VCB were assessed in univariate analyses, using the chi-square test for categorical 
Table 1 Summary of patient characteristics

\begin{tabular}{lc}
\hline Characteristics & Values \\
\hline Age (years), mean (SD) & $30.9(9.1)$ \\
Male/female & $36 / 26$ \\
Type of membrane: Bio-Gide ${ }^{\circledR} /$ Zhenghai $^{\circledR}$ & $34 / 28$ \\
Implant site: I1/I2/C & $32 / 22 / 8$ \\
Implant diameter: $3.3 \mathrm{~mm} / 4.1 \mathrm{~mm}$ & $28 / 34$ \\
Implant length: $10 \mathrm{~mm} / 12 \mathrm{~mm}$ & $24 / 38$
\end{tabular}

I1, central incisor; I2, lateral incisor; C, canine.

variables, and Student's $t$-test or the Mann-Whitney U test for continuous variables, as appropriate. Variables correlated with VCB in univariate analyses $(\mathrm{P}<0.05)$ were included in multivariable logistic regression analysis. The corresponding receiver operating characteristic (ROC) analysis was performed and the areas under curve (AUC) were calculated accordingly. Two-sided $\mathrm{P}$ values $<0.05$ was considered as indicative of statistical significance.

\section{Results}

\section{Patient characteristics}

Data of 62 patients (male: 36, female: 26) between 18 and 42 years of age were eligible to be included in the analysis. Table 1 shows the demographic features of all the patients. After a healing period of 6 months, no events of serious adverse local or systemic effects were observed in any group. All implants were stable at T1 and a second surgery was performed. No implant loss was found by the end of restoration placement.

\section{Linear changes of bone grafts}

Examination of the facial bone wall with CBCT found that all implants had a detectable facial bone wall. In the test group, the median value of TBW at T0 ranged between $2.78 \mathrm{~mm}(2.06-3.02 \mathrm{~mm})$ and $3.35 \mathrm{~mm}(2.82-4.30 \mathrm{~mm})$ at $2 \mathrm{~mm}$ and $6 \mathrm{~mm}$ apical to the platform, while the median value of TBW at T1 ranged between $2.07 \mathrm{~mm}$ $(1.80-2.51 \mathrm{~mm})$ and $2.67 \mathrm{~mm}(2.25-3.08 \mathrm{~mm})$ at $2 \mathrm{~mm}$ and $6 \mathrm{~mm}$ apical to the platform. In the control group, the corresponding value ranged between $2.36 \mathrm{~mm}(1.98$ $2.82 \mathrm{~mm})$ and $3.46 \mathrm{~mm}(2.78-4.17 \mathrm{~mm})$ at $\mathrm{T} 0$ and between $1.92 \mathrm{~mm}(1.45-2.36 \mathrm{~mm})$ and $2.60 \mathrm{~mm}(1.97-3.21 \mathrm{~mm})$ at T1, respectively (Table 2). The median value of LCB from $\mathrm{T} 0$ to $\mathrm{T} 1$ for all patients was $18.68 \%$ (2.91-36.11\%), $19.77 \%(6.45-28.84 \%)$, and $17.24 \%(4.72-33.33 \%)$ at 2,4 , and $6 \mathrm{~mm}$ level, respectively. No significant difference was found in LCB at identical measurement levels between the two groups.

\section{Volumetric changes of bone grafts}

The median value of VCB for all patients from T0 to T1 was $52.00 \%(28.30-59.80 \%)$. In the test group, the median value of VBG ranged from $386.15 \mathrm{~mm}^{3}$ (334.11-453.19) to $169.70 \mathrm{~mm}^{3}$ (118.26-301.72), while the median value of VBG ranged from $363.05 \mathrm{~mm}^{3}(289.17-573.35)$ to $221.35 \mathrm{~mm}^{3}(159.01-275.05)$ in the control group. No significant difference was found in VCB between the two groups (Table 2).

\section{Correlation analysis of morphological parameters with VCB}

Analysis of correlation by Spearman correlation test was shown in Table 3. Gender, age, type of membrane and implant site were not significantly correlated with VCB $(\mathrm{P}>0.05)$. All initial bone defect morphology-related variables except $R_{m B L / m M D}$ were correlated with VCB. mCA and $\mathrm{R}_{\mathrm{mCA} / \mathrm{mMD}}$ and $\mathrm{R}_{\mathrm{mCA} / \mathrm{mBL}}$ were positively related to $\mathrm{VCB}$ $(\mathrm{r}=0.274, \mathrm{P}=0.031 ; \mathrm{r}=0.607, \mathrm{P}<0.001 ; \mathrm{r}=0.497, \mathrm{P}<0.001$, respectively), whereas $m M D, m B L, B L_{\text {Mean }}$ and $B L_{S D}$ were negatively associated with VCB $(\mathrm{r}=-0.425, \mathrm{P}<0.001$; $\mathrm{r}=-0.380, \mathrm{P}=0.002 ; \mathrm{r}=-0.280, \mathrm{P}=0.027 ; \mathrm{r}=-0.315, \mathrm{P}=0.013$, respectively).

\section{Univariate and multivariate analysis of morphological variables with $V C B$}

The variables correlated with VCB were compared in patients classified according to the median value of VCB: $<52.00 \%$ group and $\geq 52.00 \%$ group. Differences in these variables were recorded in both groups (Table 4).

As expected, univariate analysis showed that $\mathrm{BL}_{\text {Mean }}$, $\mathrm{BL}_{\mathrm{SD}}, \mathrm{mMD}$, and $\mathrm{mBL}$ were significantly higher in the $\mathrm{VCB}$ $<52.00 \%$ group than in the VCB $\geq 52.00 \%$ group $(\mathrm{P}<0.05)$. $\mathrm{R}_{\mathrm{mCA} / \mathrm{mMD}}$ and $\mathrm{R}_{\mathrm{mCA} / \mathrm{mBL}}$ were significantly lower in the VCB $<52.00 \%$ group than in the $\mathrm{VCB} \geq 52.00 \%$ group $(\mathrm{P}<0.05)$. No significant differences were found in age, gender, type of membrane, mCA, and $\mathrm{R}_{\mathrm{mBL} / \mathrm{mMD}}$ between the two groups. For implant site, implant in canine was removed from 


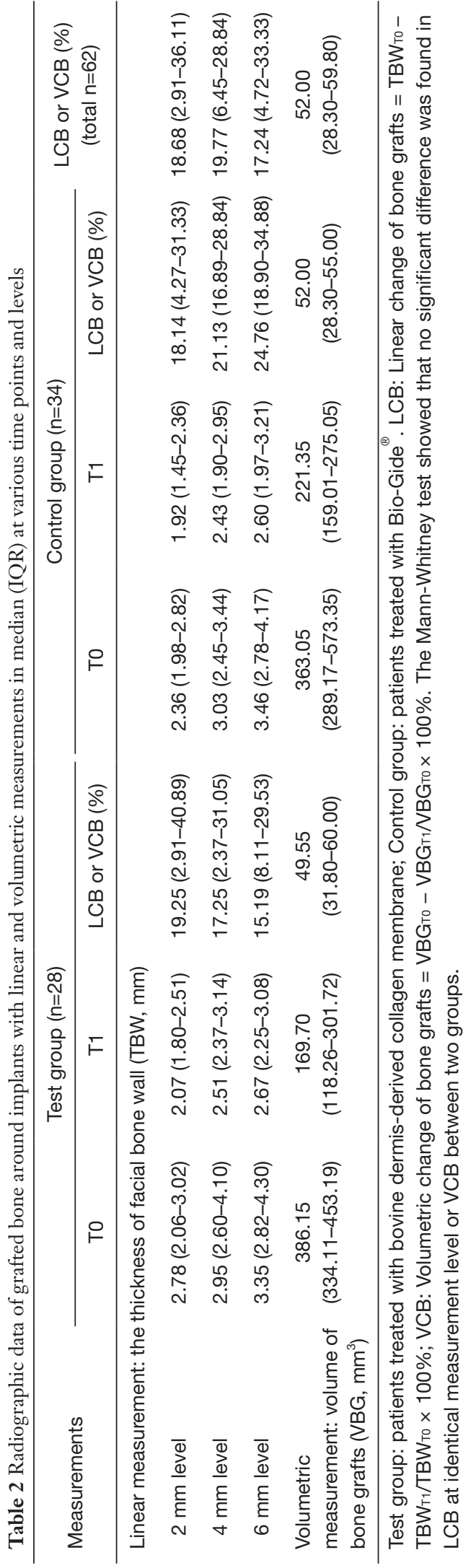

Table 3 Spearman correlation coefficients for the predictive variables and bone graft resorption rate for the total sample

\begin{tabular}{lcc}
\hline \multirow{2}{*}{ Variables } & \multicolumn{2}{c}{ Volumetric changes of bone grafts $(\%)$} \\
\cline { 2 - 3 } Gender & -0.091 & P value \\
Age & -0.088 & 0.480 \\
Membrane & -0.130 & 0.495 \\
Implant site & -0.242 & 0.312 \\
mCA & 0.274 & 0.078 \\
mMD & -0.425 & 0.031 \\
mBL & -0.380 & $<0.001$ \\
BLMean & -0.280 & 0.002 \\
BLsD & -0.315 & 0.027 \\
Ratio of $\mathrm{mCA} / \mathrm{mMD}$ & 0.607 & 0.013 \\
Ratio of $\mathrm{mCA} / \mathrm{mBL}$ & 0.497 & $<0.001$ \\
Ratio of $\mathrm{mBL} / \mathrm{mMD}$ & 0.136 & $<0.001$ \\
\hline & & 0.292 \\
\hline
\end{tabular}

univariate analysis due to the small sample size. Implant in lateral incisor was higher in the $\mathrm{VCB}<52.00 \%$ group than in the $\mathrm{VCB} \geq 52.00 \%$ group (16 vs. 6), but did not reach statistical significance.

In multivariate regression analysis including six variables: $\mathrm{BL}_{\text {Mean }}, \mathrm{BL}_{\mathrm{SD}}, \mathrm{mMD}, \mathrm{mBL}$ and $\mathrm{R}_{\mathrm{mCA} / \mathrm{mMD}}, \mathrm{R}_{\mathrm{mCA} / \mathrm{mBL}}$, only the prognosis effect of $\mathrm{BL}_{\mathrm{SD}}$ (OR: $0.220,95 \%$ CI: 0.074 to 0.655, $\mathrm{P}=0.0047$ ) and $\mathrm{R}_{\mathrm{mCA} / \mathrm{mMD}}(\mathrm{OR}: 7.045,95 \%$ CI: 2.361 to $21.024, \mathrm{P}=0.0017)$ remained significant.

\section{The potential relevance of morphological variables in the stratification of $V C B$}

To evaluate the performance of a selected morphological variable as a predictor of GBR outcome, ROC analysis for $\mathrm{BL}_{\mathrm{SD}}$ and $\mathrm{R}_{\mathrm{mCA} / \mathrm{mMD}}$, two of the primary differentiator variables of patients stratified according to the median of VCB $(52.00 \%)$ categories. ROC curve analysis showed that $\mathrm{R}_{\mathrm{mCA} / \mathrm{mMD}}$ could be used to correctly classify VCB in $78.9 \%$ patients and $\mathrm{BL}_{\mathrm{SD}}$ in $71 \%$ of patients, as classified by the median of VCB $(\geq 52.00 \%$ or $<52.00 \%)$. As shown in Figure 3, the prognostic accuracy of $\mathrm{BL}_{\mathrm{SD}}$ had an $\mathrm{AUC}$ of 0.71 (95\% CI: 0.545 to 0.883 ), with sensitivity of 0.67 and specificity of 0.75 . The ROC curve obtained using $\mathrm{R}_{\mathrm{mCA} /}$ ${ }_{m M D}$ had an AUC of 0.74 (95\% CI: 0.573 to 0.913 ), with sensitivity of 0.83 and specificity of 0.75 . 
Table 4 Baseline demographic and initial defect morphological variables are classified according to the median of VCB

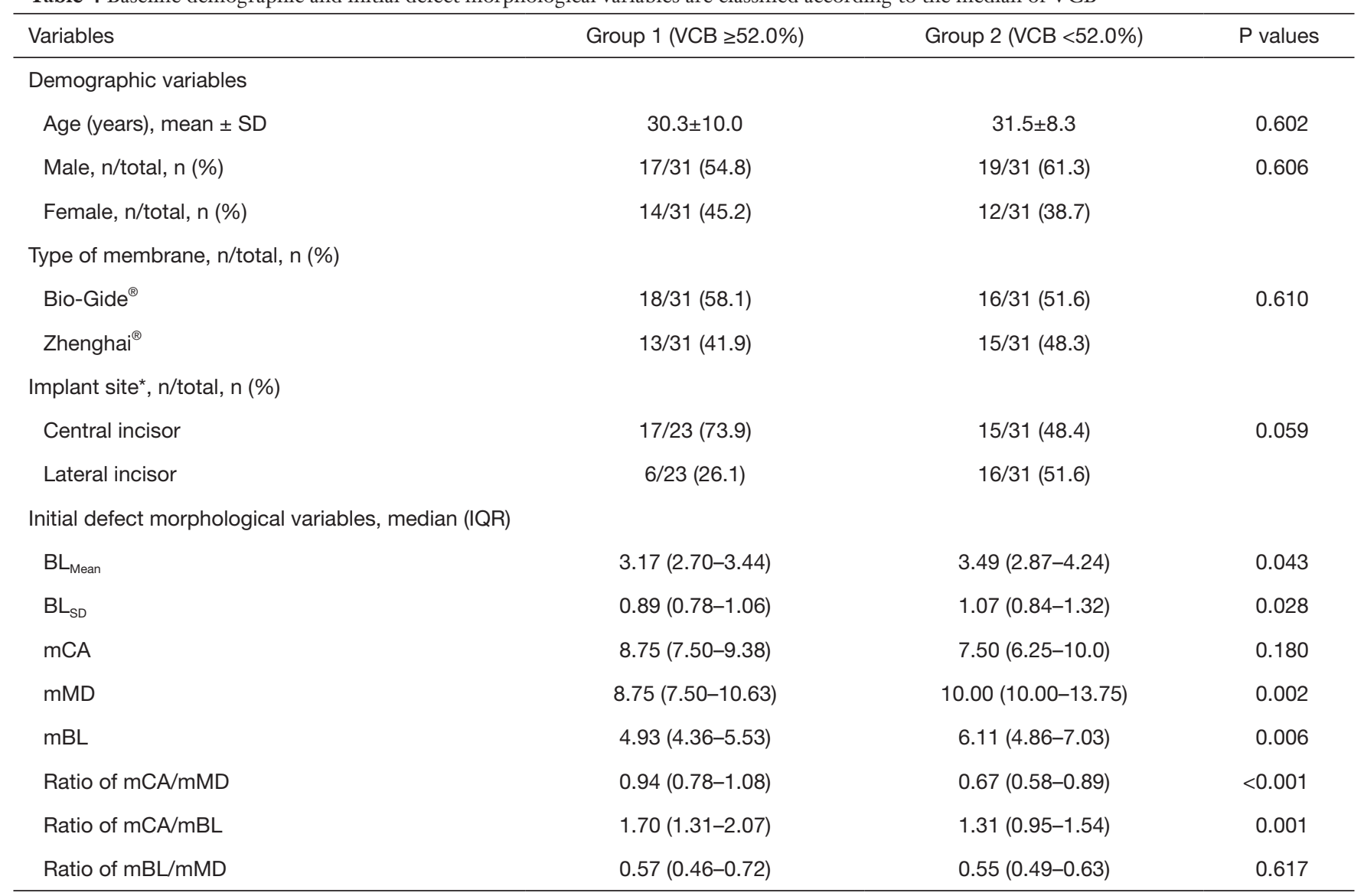

*, 8 records with canines were deleted because of the small sample size.

\section{Discussion}

The present study aimed to investigate the influence of the initial defect morphology of alveolar ridge on GBR outcomes around dental implants. The major finding of the study was two defined variables of initial defect morphology: standard deviation of buccal-lingual distance $\left(\mathrm{BL}_{\mathrm{SD}}\right)$ and ratio of maximum coronal-apical/mesial-distal distance $\left(\mathrm{R}_{\mathrm{mCA} / \mathrm{mMD}}\right)$ could be considered as morphological variables in predicting GBR outcome. As the results of ROC curve analysis, $\mathrm{R}_{\mathrm{mCA} / \mathrm{mMD}}$ could be used to correctly classify $\mathrm{VCB}$ in $78.9 \%$ and $\mathrm{BL}_{\mathrm{SD}}$ in $71 \%$ of patients according to the classification by median of VCB $(\geq 52.00 \%$ or $<52.00 \%)$.

It had been suggested that bone defect morphology could influence the regenerative outcome. It may affect the depth of the vestibule, flap tension, and subsequent wound stability after the closure of the wound. However, various influence factors can affect the GBR outcome. Thus, in this study, all patients were treated with the same bone substitute because it was found to be the compounding factor on the GBR outcome. Furthermore, all patients have received a delayed implant placement with simultaneous GBR for horizontal bone augmentation.

In this study, the GBR outcome was evaluated based on both linear and volumetric measurements of grafted bone in CBCT images. The results of linear measurements showed an acceptable outcome with over $2 \mathrm{~mm}$ facial bone thickness which achieved the goal of GBR procedures with peri-implant buccal bone thickness $\geq 1.8$ to $2 \mathrm{~mm}$ $(22,23)$. However, the results of volumetric measurements revealed the median VCB from T0 to T1 was $52.00 \%$ (28.30-59.80\%). Linear measurements only assessed the resorption rate in the mid-facial plane, while $3 \mathrm{D}$ volumetric measurements evaluated the resorption rate of the entire region of grafted bone. This might be the possible reason for the "unsatisfying" results of VCB.

Indeed, assessment of the entire area of grafted bone 


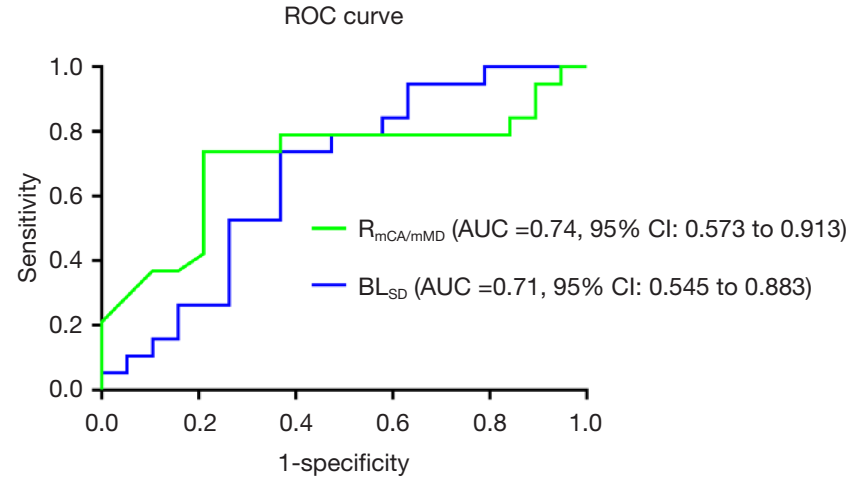

Figure 3 Morphological variables for prediction of VCB. Receiveroperating characteristic curve (ROC) analysis as assessed according to the median of VCB $(\geq 52.00 \%$ or $<52.00 \%)$. VCB, volumetric change of bone grafts; AUC, areas under curve.

allowed an intuitive observation of morphological change of grafted bone. As shown in Figure 1, bone grafts were absorbed significantly in areas away from the alveolar crest region, but the part near the alveolar crest could be retained. Soft tissue pressure and the absence of additional stabilization of barrier membrane may be the possible reasons for this result.

Our result revealed a significant negative relationship between $\mathrm{BL}_{\mathrm{SD}}$ and VCB. BL represented the buccal-lingual depth, while $\mathrm{BL}_{\mathrm{SD}}$ represented the deviation degree of buccal-lingual depth at different sites in the bone defect. That is, the larger the value, the greater the buccallingual concavity of defect. It was speculated that this type of deep defect allowed the barrier membrane to better create and maintain the space for bone regeneration. This was in accordance with previous studies that reported greater bone gain when the concavity angle of initial ridge defect increased $(24,25)$. Additionally, Tonetti et al. also demonstrated that the more contained the defect, the better the guided tissue regeneration outcome (26). This is because a better space maintenance of the barrier membrane results in a smaller risk of the barrier membrane collapsing into the defect.

Our results also showed that a significant positive relationship between $\mathrm{R}_{\mathrm{mCA} / \mathrm{mMD}}$ and $\mathrm{VCB}$. The positive correlation indicated that the more $\mathrm{mCA}$ or the less $\mathrm{mMD}$, the greater amount of bone resorption during the healing period. Intra-marrow penetration provides angiogenesis and undifferentiated mesenchymal cells that are essential for GBR (27). Bone defects in the anterior region with a greater value of $\mathrm{mMD}$ (mesial-distal distance) may allow more undifferentiated mesenchymal cells derived from the parent bone to repopulate the osseous wound space which may benefit GBR outcome. It was speculated that the significant reduction in bone gain was due to unfavorable space maintenance of barrier membrane at sites with a greater coronal-apical defect. It is worth mentioning that a fixture pin or other additional stabilizing accessory was not used in the GBR procedure for stabilization of the barrier membrane in all cases in this study. Thus, bone augmentation at sites with greater mCA may lead to higher soft tissue pressure in the augmented area and further increase the risk of the barrier membrane collapsing into the defect.

Another important result arising from this study was the fact that VCB was not affected by the type of membrane. It was because only two kinds of membranes were involved in this study, and the sample size included in this study was limited. Therefore, this result should be interpreted with caution since the membrane had a decisive influence on GBR outcome (28). In this study, age and gender were not significantly associated with VCB which was in accordance with the results of the previous study (29). In addition, implant in the lateral incisor was higher in the $\mathrm{VCB}<52.00 \%$ group than in the $\mathrm{VCB} \geq 52.00 \%$ group (16 vs. 6) though did not reach a significant difference. This may indicate that the implant site could influence the GBR outcome and further study with a large sample size would be needed.

The 3D surface rendering of the initial defect was obtained by using a mirror image of the contralateral alveolar ridge. The model could be used as a reliable model in imitating the initial ridge defect morphology which was attributed to the mature mirror technique. The method was wildly used to reconstruct symmetrically the craniomaxillofacial defect reconstruction such as mandible, condyle, and orbit (18). It is worth mentioning that object axes were defined on the basis of anatomical landmarks selected by examiners before the mirror process. The coordinate system built in the software may lead to mirrorimage confusion since involuntary patient movements during the CBCT scan could result in the midline of the coordinate system was not accordance with the midline of the patient's face.

Some limitations should be identified in this study. Firstly, the sample size and follow-up of the present study were limited. In addition, internal and external validation with a larger cohort will be needed to support the selected morphological variables for a positive prognosis. 


\section{Conclusions}

Within the limitations of this study, the present data confirmed the effect of initial ridge morphology on the GBR outcome in the anterior maxilla region. Specifically, a defect morphology with more $\mathrm{BL}_{\mathrm{SD}}$ and/or lower $\mathrm{R}_{\mathrm{mCA} / \mathrm{mMD}}$ may significantly decrease the amount of grafted bone resorption.

\section{Acknowledgments}

All authors wish to acknowledge Zhang Xiao for advice on statistical interpretation.

Funding: This work was financially supported by the Shanghai Clinical Research Center for Oral Diseases (19411950100) and the Multicenter Clinical Research Program of Shanghai Jiao Tong University School of Medicine (DLY201822).

\section{Footnote}

Reporting Checklist: The authors have completed the STROBE reporting checklist. Available at http://dx.doi. org/10.21037/atm-20-1432

Data Sharing Statement: Available at http://dx.doi. org/10.21037/atm-20-1432

Conflicts of Interest: All authors have completed the ICMJE uniform disclosure form (available at http://dx.doi. org/10.21037/atm-20-1432). JYS served as an unpaid Section Editor of Annals of Translational Medicine from October 2019 to September 2020. The authors have no other conflicts of interest to declare.

Ethical Statement: The authors are accountable for all aspects of the work in ensuring that questions related to the accuracy or integrity of any part of the work are appropriately investigated and resolved. The study conformed to the provisions of the Declaration of Helsinki (as revised in 2013). The study protocol was approved by the Ethics Committees of Shanghai Ninth People Hospitals (China, No. (2015)48], and written informed consent was obtained from the patient for publication of this study and any accompanying images.

Open Access Statement: This is an Open Access article distributed in accordance with the Creative Commons
Attribution-NonCommercial-NoDerivs 4.0 International License (CC BY-NC-ND 4.0), which permits the noncommercial replication and distribution of the article with the strict proviso that no changes or edits are made and the original work is properly cited (including links to both the formal publication through the relevant DOI and the license). See: https://creativecommons.org/licenses/by-nc-nd/4.0/.

\section{References}

1. Bassetti MA, Bassetti RG, Bosshardt DD. The alveolar ridge splitting/expansion technique: a systematic review. Clin Oral Implants Res 2016;27:310-24.

2. Dasmah A, Thor A, Ekestubbe A, et al. Marginal bonelevel alterations at implants installed in block versus particulate onlay bone grafts mixed with platelet-rich plasma in atrophic maxilla. a prospective 5-year followup study of 15 patients. Clin Implant Dent Relat Res 2013;15:7-14.

3. Nogueira RLM, Osterne RLV, Abreu RT, et al. Alternative Distraction Osteogenesis Technique After Implant Placement for Alveolar Ridge Augmentation of the Maxilla. J Oral Maxillofac Surg 2017;75:1402.e1-8.

4. Chiapasco M, Zaniboni M. Clinical outcomes of GBR procedures to correct peri-implant dehiscences and fenestrations: a systematic review. Clin Oral Implants Res 2009;20 Suppl 4:113-23.

5. Benic GI, Hammerle CH. Horizontal bone augmentation by means of guided bone regeneration. Periodontol 2000 2014;66:13-40.

6. Meloni SM, Jovanovic SA, Pisano M, et al. One-stage horizontal guided bone regeneration with autologous bone, anorganic bovine bone and collagen membranes: Follow-up of a prospective study 30 months after loading. Eur J Oral Implantol 2018;11:89-95.

7. Esposito M, Grusovin MG, Felice P, et al. The efficacy of horizontal and vertical bone augmentation procedures for dental implants - a Cochrane systematic review. Eur J Oral Implantol 2009;2:167-84.

8. von Arx T, Kurt B. Implant placement and simultaneous ridge augmentation using autogenous bone and a micro titanium mesh: a prospective clinical study with 20 implants. Clin Oral Implants Res 1999;10:24-33.

9. Urban IA, Monje A, Lozada JL, et al. Longterm Evaluation of Peri-implant Bone Level after Reconstruction of Severely Atrophic Edentulous Maxilla via Vertical and Horizontal Guided Bone Regeneration 
in Combination with Sinus Augmentation: A Case Series with 1 to 15 Years of Loading. Clin Implant Dent Relat Res 2017;19:46-55.

10. Carpio L, Loza J, Lynch S, et al. Guided bone regeneration around endosseous implants with anorganic bovine bone mineral. A randomized controlled trial comparing bioabsorbable versus non-resorbable barriers. J Periodontol 2000;71:1743-9.

11. Zitzmann NU, Naef R, Scharer P. Resorbable versus nonresorbable membranes in combination with BioOss for guided bone regeneration. Int J Oral Maxillofac Implants 1997;12:844-52.

12. Merli M, Moscatelli M, Mariotti G, et al. Comparing membranes and bone substitutes in a one-stage procedure for horizontal bone augmentation. Three-year postloading results of a double-blind randomised controlled trial. Eur J Oral Implantol 2018;11:441-52.

13. Schwarz F, Herten M, Ferrari D, et al. Guided bone regeneration at dehiscence-type defects using biphasic hydroxyapatite + beta tricalcium phosphate (Bone Ceramic) or a collagen-coated natural bone mineral (BioOss Collagen): an immunohistochemical study in dogs. Int J Oral Maxillofac Surg 2007;36:1198-206.

14. Strietzel FP, Khongkhunthian P, Khattiya R, et al. Healing pattern of bone defects covered by different membrane types--a histologic study in the porcine mandible. J Biomed Mater Res B Appl Biomater 2006;78:35-46.

15. Misch CE, Judy KW. Classification of partially edentulous arches for implant dentistry. Int J Oral Implantol 1987;4:7-13.

16. Wang HL, Al-Shammari K. HVC ridge deficiency classification: a therapeutically oriented classification. Int J Periodontics Restorative Dent 2002;22:335-43.

17. Khojasteh A, Motamedian SR, Sharifzadeh N, et al. The influence of initial alveolar ridge defect morphology on the outcome of implants in augmented atrophic posterior mandible: an exploratory retrospective study. Clin Oral Implants Res 2017;28:e208-17.

18. Oh JH. Recent advances in the reconstruction of craniomaxillofacial defects using computer-aided design/ computer-aided manufacturing. Maxillofac Plast Reconstr Surg 2018;40:2.

19. Benic GI, Elmasry M, Hammerle CH. Novel digital imaging techniques to assess the outcome in oral rehabilitation with dental implants: a narrative review. Clin Oral Implants Res 2015;26 Suppl 11:86-96.

20. Pelekos G, Tse JMN, Ho D, et al. Defect Morphology,
Bone Thickness, Exposure Settings and Examiner Experience Affect the Diagnostic Accuracy of Standardized Digital Periapical Radiographic Images but not of Cone Beam Computed Tomography in the Detection of PeriImplant Osseous Defects: an in Vitro Study. J Clin Periodontol 2019;46:1294-302.

21. Li Y, Qiao SC, Gu YX, et al. A novel semiautomatic segmentation protocol to evaluate guided bone regeneration outcomes: A pilot randomized, controlled clinical trial. Clin Oral Implants Res 2019;30:344-52.

22. Qahash M, Susin C, Polimeni G, et al. Bone healing dynamics at buccal peri-implant sites. Clin Oral Implants Res 2008;19:166-72.

23. Miyamoto Y, Obama T. Dental cone beam computed tomography analyses of postoperative labial bone thickness in maxillary anterior implants: comparing immediate and delayed implant placement. Int J Periodontics Restorative Dent 2011;31:215-25.

24. Garaicoa C, Suarez F, Fu JH, et al. Using Cone Beam Computed Tomography Angle for Predicting the Outcome of Horizontal Bone Augmentation. Clin Implant Dent Relat Res 2015;17:717-23.

25. Park SH, Brooks SL, Oh TJ, et al. Effect of ridge morphology on guided bone regeneration outcome: conventional tomographic study. J Periodontol 2009;80:1231-6.

26. Tonetti MS, Pini-Prato G, Cortellini P. Periodontal regeneration of human intrabony defects. IV. Determinants of healing response. J Periodontol 1993;64:934-40.

27. Nishimura I, Shimizu Y, Ooya K. Effects of cortical bone perforation on experimental guided bone regeneration. Clin Oral Implants Res 2004;15:293-300.

28. Retzepi M, Donos N. Guided Bone Regeneration: biological principle and therapeutic applications. Clin Oral Implants Res 2010;21:567-76.

29. Phillips DJ, Swenson DT, Johnson TM. Buccal bone thickness adjacent to virtual dental implants following guided bone regeneration. J Periodontol 2019;90:595-607.

Cite this article as: Li Y, Zhang XM, Qian SJ, Qiao SC, Lai HC, Shi JY. The influence of initial defect morphology of alveolar ridge on volumetric change of grafted bone following guided bone regeneration in the anterior maxilla region: an exploratory retrospective study. Ann Transl Med 2020;8(23):1592. doi: 10.21037/atm-20-1432 